\title{
Nontrivial solutions of inverse discrete problems with sign-changing nonlinearities
}

\author{
Alberto Cabada ${ }^{1 *}$ (D) and Nikolay D. Dimitrov²
}

\author{
"Correspondence: \\ alberto.cabada@usc.es \\ 'Departamento de Estatística, \\ Análise Matemática e Optimización, \\ Instituto de Matemáticas, Facultade \\ de Matemáticas, Universidade de \\ Santiago de Compostela, Santiago \\ de Compostela, Spain \\ Full list of author information is \\ available at the end of the article
}

\begin{abstract}
This paper is concerned with the existence of solutions of an inverse discrete problem with sign-changing nonlinearity. This kind of problems includes, as a particular case, $n$th order difference equations coupled with suitable conditions on the boundary of the interval of definition. It would be valid for the case in which the related Green's function is positive on a subset of its rectangle of definition.

The existence results follow from spectral theory, as an application of the Krein-Rutman theorem and by means of degree theory.
\end{abstract}

MSC: 39A10; 39A06; 39A70

Keywords: Inverse discrete problem; Difference equation; Green's function; Spectral radius

\section{Introduction}

During the last years, many authors discussed the existence of solutions for boundary value problems by using various topological methods. However, there are just a few results with sign-changing nonlinearities. Usually, in order to obtain positive solutions of semipositone problems for ordinary differential or difference equations, by using fixed point methods, the nonlinearity terms need to be bounded from below and ultimately positive. For example, in [2] the authors studied the following problem:

$$
\left\{\begin{array}{l}
\left(p(t) u^{\prime}\right)^{\prime}+\lambda g(t, u)=0, \quad t \in(a, b), \\
\gamma_{1} u(a)-\gamma_{2} p(a) u^{\prime}(a)=0, \quad \gamma_{3} u(b)-\gamma_{4} p(b) u^{\prime}(b)=0,
\end{array}\right.
$$

where $g:[a, b] \times \mathbb{R}^{+} \rightarrow \mathbb{R}$ is continuous, bounded from below (i.e., $g(t, z)+M>0$ for some $M>0)$ and $\lim _{z \rightarrow \infty} \frac{f(t, z)}{z}=\infty$ uniformly for $t \in[\alpha, \beta] \subset(a, b)$. Later, Bai and Xu [4] discussed its discrete analog and it also required boundedness from below on the nonlinearity term, as well as a superlinear condition at $\infty$. We also refer the reader to $[9,11,12]$, where the authors used topological methods to deduce, under a similar hypothesis, the existence results to a discrete fractional semipositone boundary value problem.

By using Guo-Krasnosel'skii's fixed point theorem, Bai, Henderson, and Zeng [3] obtained the existence of positive solutions of the discrete Neumann boundary value prob-

(c) The Author(s) 2019. This article is distributed under the terms of the Creative Commons Attribution 4.0 International License (http://creativecommons.org/licenses/by/4.0/), which permits unrestricted use, distribution, and reproduction in any medium, provided you give appropriate credit to the original author(s) and the source, provide a link to the Creative Commons license, and indicate if changes were made. 
lem

$$
\left\{\begin{array}{l}
-\Delta^{2} u(k-1)=f(k, u(k)), \quad k \in[1, T]_{\mathbb{Z}} \\
0=\Delta u(0)=\Delta u(T)
\end{array}\right.
$$

where the nonlinearity term $f:[1, T]_{\mathbb{Z}} \times \mathbb{R}^{+} \rightarrow \mathbb{R}$ is a continuous sign-changing function, for which there exist a function $h:[1, T]_{\mathbb{Z}} \rightarrow \mathbb{R}^{+}$, with $h \neq 0$ on $[1, T]_{\mathbb{Z}}$, and a constant $L>0$ such that $f(t, z)+L z+h(t) \geq 0,(t, z) \in[1, T]_{\mathbb{Z}} \times \mathbb{R}^{+}$.

In [17], using Krein-Rutman theorem, Zhang studied the fourth order singular boundary value problem

$$
\left\{\begin{array}{l}
u^{(4)}(t)=h(t) f(u(t)), \quad t \in(0,1) \\
0=u(0)=u(1)=u^{\prime}(0)=u^{\prime}(1)
\end{array}\right.
$$

under some suitable conditions concerning the first characteristic value corresponding to the relevant linear operator. Here, $h$ is allowed to be singular at both $t=0$ and $t=1$. In particular, $f: \mathbb{R} \rightarrow \mathbb{R}$ may be a sign-changing and unbounded function from below, and the existence of a control from below function related to $f$ is not assumed. The existence results of nontrivial solutions and positive-negative solutions are given by the topological degree theory and the fixed point index theory, respectively.

A similar idea can be found in a very recent paper [18], where under suitable conditions concerning the first eigenvalue corresponding to the relevant linear problem, the authors established the existence of nontrivial solutions for boundary value problems of the following fourth order difference equation with a sign-changing nonlinearity:

$$
\left\{\begin{array}{l}
\Delta^{4} u(k-2)=f(k, u(k)), \quad k \in[2, T]_{\mathbb{Z}} \\
0=u(1)=u(T+1)=\Delta^{2} u(0)=\Delta^{2} u(T)
\end{array}\right.
$$

where $T \geq 5$ is an integer and $f:[2, T]_{\mathbb{Z}} \times \mathbb{R} \rightarrow \mathbb{R}$ is a continuous function. The results are based on the topological degree theory and generalize some previous results obtained for this problem. It is important to point out that our results generalize some ones given in that reference, in fact, as we will see in Example 9, Theorems 3.2 and 3.1 in [18] are particular cases (respectively) of Theorems 5 and 6 in this work.

Our aim is to extend these results as we study the following $n$th order boundary value problem:

$$
(P) \quad\left\{\begin{array}{l}
u(k+n)+\sum_{i=0}^{n-1} p_{i}(k) u(k+i)=f(k, u(k)), \quad k \in I \equiv[a, b]_{\mathbb{Z}} \\
L_{i} u=0, \quad i=1, \ldots, n
\end{array}\right.
$$

Here, $f: I \times \mathbb{R} \rightarrow \mathbb{R}$ is a continuous function, $b-a \geq 2$, and

$$
L_{i}: \mathbb{R}^{b-a+1} \rightarrow \mathbb{R}, \quad i=1, \ldots, n,
$$

are linear operators, for which the following conditions for the related Green's function $G(k, s)$ are fulfilled: 
(G1) $G(k, s) \geq 0$ for all $k \in I$ and $s \in I$.

(G2) $G(k, s)$ is symmetric for all $k \in I$ and $s \in I$, i.e., $G(k, s)=G(s, k)$.

(G3) There is $k_{0} \in I$ such that $G\left(k_{0}, s\right)>0$ for all $s \in I$.

Remark 1 Notice that condition (G2) implies that all the eigenvalues of the matrix $(G(k, s))_{k, s \in I}$ are real and that its associated Jordan matrix is diagonal. As a consequence, all its eigenvalues are zero if and only if $G$ is identically zero which, from (G3), is not true. So we deduce that the spectral radius is strictly positive.

There are several papers in the literature, where the related Green's function of the studied difference equation verifies previous properties for a suitable value of parameters, see, for instance, $[1,6,7]$ (and in [5] for order four), where the $n$th order Problem $(P)$ with periodic conditions, i.e.,

$$
L_{i} x:=x(i-1)-x(N+i-1), \quad i=1, \ldots, n,
$$

has been studied.

The Neumann conditions were considered in [8].

So, with this idea in mind, we study a more general problem that includes the difference equations as particular cases. That is, we consider the general inverse discrete problem

$$
u(k):=\sum_{s=a}^{b} G(k, s) f(s, u(s)), \quad k \in I,
$$

where $G$ satisfies conditions (G1)-(G3), but it is not necessarily the Green's function of a related linear operator.

To deduce the existence results of equation (2), we will work with spectral and degree theory. To this end, define the space $E$ as the collection of all maps from $I$ to $\mathbb{R}$ equipped with the norm $\|u\|=\max _{k \in I}|u(k)|$. Clearly, $E$ is a Banach space.

Now, we recall some definitions that will be useful in the sequel. Let $X$ be a Banach space. We say that $K \subset X$ is a cone if $K$ is a closed convex set such that $\lambda K \subset K$ for all $\lambda \geq 0$ and $K \cap(-K)=\{0\}$. If $\overline{K-K}=X$, i.e., the set $\{u-v \mid u, v \in K\}$ is dense in $X$, then $K$ is called a total cone. If $K-K=X, K$ is called a reproducing cone. If a cone has nonempty interior $K^{0}$, then it is called a solid cone. Any solid cone has the property that $K-K=X$; in particular, it is total.

Let us recall Krein-Rutman theorem.

Theorem 2 (Krein-Rutman, [10, Theorem 19.2]) Let $X$ be a Banach space, $K \subset X$ be a total cone, and $T: X \rightarrow X$ be a compact linear operator that is positive $(i . e ., T(K) \subset K)$ with positive spectral radius $r(T)$. Then $r(T)$ is an eigenvalue with an eigenvector $u \in K \backslash\{0\}$, i.e., $T u=r(T) u$.

Now we introduce two useful results using the properties of the topological degree, which can be found in [13]. 
Lemma 3 Let $X$ be a Banach space and $\Omega$ be a bounded open set in X. Suppose that A : $\Omega \rightarrow X$ is a continuous compact operator. If there exists $u_{0} \in X \backslash\{0\}$ such that

$$
u-A u \neq \mu u_{0} \quad \text { for all } u \in \partial \Omega \text { and } \mu \geq 0,
$$

then the topological degree $\operatorname{deg}(I-A, \Omega, 0)=0$.

Lemma 4 Let $X$ be a Banach space and $\Omega$ be a bounded open set in $X$ with $0 \in \Omega$. Suppose that $A: \Omega \rightarrow X$ is a continuous compact operator. If

$$
A u \neq \mu u \text { for all } u \in \partial \Omega \text { and } \mu \geq 1,
$$

then the topological degree $\operatorname{deg}(I-A, \Omega, 0)=1$.

The paper is scheduled as follows: In the next section we deduce the main properties of the kernel G. Section 3 is devoted to nonlinear problem (2). In it, under suitable assumptions on the nonlinearity $f$, which is allowed to change its sign, related to the first eigenvalue of the linear problem, the existence of a nontrivial solution is proved. The paper ends with some examples where the applicability of the results is pointed out.

\section{Kernel properties}

In this section we present some additional assumptions on the kernel $G$, and we deduce some suitable properties.

Define the cone $K$ on $E$ as follows:

$$
K:=\{u \in E: u(k) \geq 0, k \in I\} .
$$

To the end of the paper, let $B_{\rho}=\{u \in E:\|u\|<\rho\}$ for $\rho>0, \partial B_{\rho}=\{u \in E:\|u\|=\rho\}$, and $\overline{B_{\rho}}=\{u \in E:\|u\| \leq \rho\}$.

Define the operator $T: E \rightarrow E$ as follows:

$$
T u(k):=\sum_{s=a}^{b} G(k, s) f(s, u(s)), \quad k \in I .
$$

By definition, we have that the solutions of problem (2) coincide with the fixed points of operator $T$.

Now, define the operator

$$
(L u)(k):=\sum_{s=a}^{b} G(k, s) u(s), \quad k \in I .
$$

From Remark 1 , we have that $r(L)$, the spectral radius of operator $L$, is such that $r(L)>0$. Thus, since the compactness and the continuity properties are equivalent on finite dimensional spaces, we can apply Krein-Rutman theorem to operator $L$. So, it follows that

$$
\sum_{s=a}^{b} G(k, s) v(s)=r(L) v(k)
$$

where $v(k) \geq 0$ on $I, v \neq \equiv$ on $I$, is the corresponding eigenfunction. 
Notice that if $v\left(k_{0}\right)=0$, from condition (G3) we arrive at the following contradiction:

$$
0<\sum_{s=a}^{b} G\left(k_{0}, s\right) v(s)=r(L) v\left(k_{0}\right)=0 .
$$

Moreover, since $G$ is symmetric, we have

$$
\sum_{k=a}^{b}\left(\sum_{s=a}^{b} G(k, s) u(s)\right) v(k)=\sum_{k=a}^{b}\left(\sum_{s=a}^{b} G(k, s) v(s)\right) u(k) .
$$

In the sequel, we introduce the following functions:

$$
l_{1}(k):=\min _{\substack{\sigma \in I, s \in I \\ G(\sigma, s) \neq 0}} \frac{G(k, s)}{G(\sigma, s)}, \quad k \in I,
$$

and

$$
\varphi(s):=\max _{\sigma \in I}\{G(\sigma, s)\}, \quad s \in I .
$$

It is clear, from (G1), that $l_{1} \geq 0$ on $I$ and, by condition (G3), that $l_{1}\left(k_{0}\right)>0$. From (G1) and (G3) we have that $\varphi(s)>0$ for all $s \in I$. Moreover, the following inequalities hold:

$$
l_{1}(k) G(\sigma, s) \leq G(k, s) \leq \varphi(s) \text { for all } k, s, \sigma \in I .
$$

\section{Nonlinear problem}

This section is devoted to proving the existence of a nontrivial solution of Problem (2) which, as we have noted in previous sections, is equivalent to finding a fixed point of operator $T$ defined on (3). The proofs follow similar arguments to the ones developed by Zhang, O'Regan, and $\mathrm{Fu}$ in [18] for equation (1).

So, we present some assumptions about the nonlinearity $f$ :

(H0) $f: I \times \mathbb{R} \rightarrow \mathbb{R}$ is a continuous function on $I \times \mathbb{R}$.

(H1) $\lim _{u \rightarrow 0^{+}} \inf \frac{f(k, u)}{u}>\frac{1}{r(L)}, \lim _{u \rightarrow 0^{-}} \sup \frac{f(k, u)}{u}<\frac{1}{r(L)}$ for all $k \in I$.

(H2) $\lim _{|u| \rightarrow \infty} \sup \frac{|f(k, u)|}{|u|}<\frac{1}{r(L)}$ for all $k \in I$.

Our first main result is as follows.

Theorem 5 Suppose that (H0)-(H2) hold. Then Problem (2) has at least one nontrivial solution.

Proof From $(H 1)$ there exist $\varepsilon_{0} \in\left(0, \frac{1}{r(L)}\right)$ and $r>0$ such that

$$
f(k, u) \geq\left(\frac{1}{r(L)}+\varepsilon_{0}\right) u \quad \text { for all } u \in[0, r] \text { and } k \in I
$$

and

$$
f(k, u) \geq\left(\frac{1}{r(L)}-\varepsilon_{0}\right) u \quad \text { for all } u \in[-r, 0] \text { and } k \in I
$$


Using the above two inequalities, one can obtain that

$$
f(k, u) \geq\left(\frac{1}{r(L)}+\varepsilon_{0}\right) u \quad \text { for all } u \in[-r, r] \text { and } k \in I
$$

and

$$
f(k, u) \geq\left(\frac{1}{r(L)}-\varepsilon_{0}\right) u \quad \text { for all } u \in[-r, r] \text { and } k \in I
$$

Now, taking into account that

$$
\delta:=\sum_{k=a}^{b} l_{1}(k) v(k) \geq l_{1}\left(k_{0}\right) v\left(k_{0}\right)>0,
$$

we introduce the following cone:

$$
K_{1}:=\left\{u \in K \mid \sum_{k=a}^{b} u(k) v(k) \geq \delta\|u\|\right\} .
$$

We claim that $L(K) \subset K_{1}$. Indeed, using the definition of $L$, for all $u \in K, L u \geq 0$ on $I$. So, for any $\sigma \in I$, we have that

$$
\begin{aligned}
\sum_{k=a}^{b}(L u)(k) v(k) & =\sum_{k=a}^{b}\left(\sum_{s=a}^{b} G(k, s) u(s)\right) v(k) \\
& \geq \sum_{k=a}^{b}\left(l_{1}(k) \sum_{s=a}^{b} G(\sigma, s) u(s)\right) v(k) \\
& =\left(\sum_{k=a}^{b} l_{1}(k) v(k)\right)(L u)(\sigma) .
\end{aligned}
$$

Thus,

$$
\sum_{k=a}^{b}(L u)(k) v(k) \geq \delta\|L u\|
$$

Notice that, since $v=\frac{L v}{r(L)}$ it follows that $v \in K_{1}$.

Now, we claim that $u-T u \neq \mu \nu$ for all $u \in \partial B_{r}$ and $\mu \geq 0$. Assume, on the contrary, that there exist $u_{1} \in \partial B_{r}$ and $\mu_{1} \geq 0$ such that

$$
u_{1}-T u_{1}=\mu_{1} v
$$

Using (10) and (12), we have that

$$
u_{1}(k) \geq\left(\frac{1}{r(L)}+\varepsilon_{0}\right) \sum_{s=a}^{b} G(k, s) u_{1}(s)=\left(\frac{1}{r(L)}+\varepsilon_{0}\right) L u_{1}(k), \quad k \in I .
$$


Multiplying both sides of the above inequality by $v(k)$, summing from $a$ to $b$, and using (5) and (6), we obtain that

$$
\begin{aligned}
\sum_{k=a}^{b} u_{1}(k) v(k) & \geq\left(\frac{1}{r(L)}+\varepsilon_{0}\right) \sum_{k=a}^{b}\left(\sum_{s=a}^{b} G(k, s) u_{1}(s)\right) v(k) \\
& =\left(\frac{1}{r(L)}+\varepsilon_{0}\right) \sum_{k=a}^{b}\left(\sum_{s=a}^{b} G(k, s) v(s)\right) u_{1}(k) \\
& =\left(1+\varepsilon_{0} r(L)\right) \sum_{k=a}^{b} u_{1}(k) v(k),
\end{aligned}
$$

whence $\sum_{k=a}^{b} u_{1}(k) v(k) \leq 0$.

On the other hand, from (12) we have, for any $k \in I$,

$$
\begin{aligned}
& u_{1}(k)-\left(\frac{1}{r(L)}-\varepsilon_{0}\right)\left(L u_{1}\right)(k) \\
& =\left(T u_{1}\right)(k)-\left(\frac{1}{r(L)}-\varepsilon_{0}\right)\left(L u_{1}\right)(k)+\mu_{1} v(k) \\
& =\sum_{s=a}^{b} G(k, s) f\left(s, u_{1}(s)\right)-\left(\frac{1}{r(L)}-\varepsilon_{0}\right) \sum_{s=a}^{b} G(k, s) u_{1}(s)+\mu_{1} v(k) \\
& =\sum_{s=a}^{b} G(k, s)\left(f\left(s, u_{1}(s)\right)-\left(\frac{1}{r(L)}-\varepsilon_{0}\right) u_{1}(s)\right)+\mu_{1} v(k) .
\end{aligned}
$$

Now, using the latter together with $L(K) \subset K_{1}, v \in K_{1}$, and (11), gives us that $u_{1}-\left(\frac{1}{r(L)}-\right.$ $\left.\varepsilon_{0}\right) L u_{1} \in K_{1}$.

Hence, from (5) and (6), we deduce

$$
\begin{aligned}
\left\|u_{1}-\left(\frac{1}{r(L)}-\varepsilon_{0}\right) L u_{1}\right\| & \leq \frac{1}{\delta} \sum_{k=a}^{b}\left(u_{1}(k)-\left(\frac{1}{r(L)}-\varepsilon_{0}\right)\left(L u_{1}\right)(k)\right) v(k) \\
& =\frac{1}{\delta}\left(\sum_{k=a}^{b} u_{1}(k) v(k)-\left(\frac{1}{r(L)}-\varepsilon_{0}\right) \sum_{k=a}^{b} r(L) u_{1}(k) v(k)\right) \\
& =\frac{r(L) \varepsilon_{0}}{\delta} \sum_{k=a}^{b} u_{1}(k) v(k) \\
& \leq 0 .
\end{aligned}
$$

The above inequality implies that

$$
u_{1}=\left(\frac{1}{r(L)}-\varepsilon_{0}\right) L u_{1},
$$

which, together with $\left(\frac{1}{r(L)}-\varepsilon_{0}\right)^{-1}>r(L)$, implies that $u_{1}=0$, which contradicts the fact that $u_{1} \in \partial B_{r}$. 
Thus, (12) is false and, from Lemma 3, it follows that

$$
\operatorname{deg}\left(I-T, B_{r}, 0\right)=0 .
$$

From $(H 2)$ there exist $\varepsilon_{1} \in\left(0, \frac{1}{r(L)}\right)$ and $c_{1}>0$ such that

$$
|f(k, u)| \leq\left(\frac{1}{r(L)}-\varepsilon_{1}\right)|u|+c_{1} \quad \text { for all } k \in I \text { and } u \in \mathbb{R}
$$

Let

$$
X:=\{u \in E \mid u=\lambda T u, \lambda \in[0,1]\} .
$$

Now, we will show that $X$ is bounded in $E$.

To this end, as a direct consequence of [16, (57d)], we have that for any $0<\alpha<\frac{1}{r(L)}$, its corresponding Neumann series converges in the operator norm:

$$
(I-\alpha L)^{-1}=\sum_{n=0}^{\infty}(\alpha L)^{n}
$$

In addition, since it is a sum of positive operators, we have that $(I-\alpha L)^{-1}$ is a positive operator on $E$.

On the other hand, using (14), we have that the following inequalities are fulfilled for all $u \in X$ :

$$
\begin{aligned}
|u(k)| & =\lambda|(T u)(k)| \leq \sum_{s=a}^{b} G(k, s)|f(s, u(s))| \\
& \leq \sum_{s=a}^{b} G(k, s)\left(\left(\frac{1}{r(L)}-\varepsilon_{1}\right)|u(s)|+c_{1}\right) .
\end{aligned}
$$

Clearly, the previous inequality is of the form $|u| \leq \alpha L|u|+v$, with $0<\alpha=\frac{1}{r(L)}-\varepsilon_{1}<\frac{1}{r(L)}$ and $v=\sum_{s=a}^{b} G(k, s) c_{1}$.

As a consequence, from the positiveness of $(I-\alpha L)^{-1}$ on $E$, we deduce that $|u| \leq(I-$ $\alpha L)^{-1} v, u$ is bounded, i.e., $X$ is bounded.

Now, one can choose $R>\max \left\{\sup _{u \in X}\|u\|, r\right\}$ for $r$ defined by (10). Then, $\lambda T u \neq u$ for all $u \in \partial B_{R}$ and $\lambda \in[0,1]$. From Lemma 4 it follows that $\operatorname{deg}\left(I-T, B_{R}, 0\right)=1$.

Using the latter one, together with $\operatorname{deg}\left(I-T, B_{r}, 0\right)=0$, gives us

$$
\operatorname{deg}\left(I-T, B_{R} \backslash \overline{B_{r}}, 0\right)=\operatorname{deg}\left(I-T, B_{R}, 0\right)-\operatorname{deg}\left(I-T, B_{r}, 0\right)=1 .
$$

Therefore, operator $T$ has at least one fixed point in $B_{R} \backslash \overline{B_{r}}$, which is a nontrivial solution of (2).

Before formulating our second main result, let us introduce the following conditions about the nonlinearity: 
(H3) There exist two constants $a_{1}>0, b_{1}>0$ and a function $\Phi \in C\left(\mathbb{R}, \mathbb{R}^{+}\right)$such that

$$
f(k, u) \geq-a_{1}-b_{1} \Phi(u) \quad \text { for all } u \in \mathbb{R} \text { and } k \in I
$$

(H4) $\lim _{|u| \rightarrow+\infty} \frac{\Phi(u)}{|u|}=0$.

(H5) $\lim _{|u| \rightarrow+\infty} \inf \frac{f(k, u)}{|u|}>\frac{1}{r(L)}$ for all $k \in I$.

(H6) $\lim _{|u| \rightarrow 0} \sup \frac{|f(k, u)|}{|u|}<\frac{1}{r(L)}$ for all $k \in I$.

The result is the following.

Theorem 6 Suppose that $(H 0)$ and (H3)-(H6) hold. Then Problem (2) has at least one nontrivial solution.

Proof From $(H 5)$ there exist $\varepsilon_{2}>0$ and $N_{1}>0$ such that

$$
f(k, u) \geq\left(\frac{1}{r(L)}+\varepsilon_{2}\right)|u| \quad \text { for all } k \in I \text { and }|u|>N_{1} \text {. }
$$

For any given $\varepsilon_{2}>b_{1} \varepsilon_{3}$, using $(H 4)$, there exists $N_{2}>N_{1}$ such that

$$
\Phi(u) \leq \varepsilon_{3}|u| \quad \text { for all } u \text { with }|u|>N_{2} \text {. }
$$

From $(H 3)$, since $a_{1}>0, b_{1}>0$ and $\Phi$ is a nonnegative function, we have

$$
f(k, u) \geq\left(\frac{1}{r(L)}+\varepsilon_{2}\right)|u|-a_{1}-b_{1} \Phi(u) \geq\left(\frac{1}{r(L)}+\varepsilon_{2}\right)|u|-a_{1}-b_{1} \varepsilon_{3}|u|
$$

for all $u$ with $|u|>N_{2}$.

Let us denote $c_{2}=\left(\frac{1}{r(L)}+\varepsilon_{2}-b_{1} \varepsilon_{3}\right) N_{2}+\max _{k \in I,|u| \leq N_{2}}|f(k, u)|, c_{3}=c_{2}+a_{1}$, and $\Phi^{*}=$ $\max _{|u| \leq N_{2}} \Phi(u)$. Then

$$
f(k, u) \geq\left(\frac{1}{r(L)}+\varepsilon_{2}-b_{1} \varepsilon_{3}\right)|u|-c_{3} \quad \text { for all } k \in I \text { and } u \in \mathbb{R} \text {. }
$$

Note that $\varepsilon_{3}$ can be chosen arbitrarily small, and let us set

$$
\begin{aligned}
& R_{1}=\frac{\left(c_{3}+b_{1} \Phi^{*}\right) \max _{k \in I} l_{1}(k) \sum_{s=a}^{b} \varphi(s)}{1-b_{1} \varepsilon_{3} \max _{k \in I} l_{1}(k) \sum_{s=a}^{b} \varphi(s)} \\
& R_{2}=\frac{\left(c_{3}+b_{1} \Phi^{*}\right)\left(\left(\varepsilon_{2}-b_{1} \varepsilon_{3}\right)\left(1+\max _{k \in I} l_{1}(k)\right)+\frac{1}{r(L)}\right) \sum_{s=a}^{b} \varphi(s)}{\left(\varepsilon_{2}-b_{1} \varepsilon_{3}\right)\left(1-b_{1} \varepsilon_{3} \max _{k \in I} l_{1}(k) \sum_{s=a}^{b} \varphi(s)\right)-\left(\frac{1}{r(L)}+\varepsilon_{2}-b_{1} \varepsilon_{3}\right) b_{1} \varepsilon_{3} \sum_{s=a}^{b} \varphi(s)},
\end{aligned}
$$

and

$$
R>\max \left\{R_{1}, R_{2}\right\} .
$$

Now, we will prove that

$$
u-T u \neq \mu \nu \quad \text { for every } u \in \partial B_{R} \text { and } \mu>0 \text {. }
$$


Notice that, if the equality holds for $\mu=0$, we have proved the existence of the fixed point of $T$.

Define the cone $K_{2}$ as follows:

$$
K_{2}:=\left\{u \in K: u(k) \geq l_{1}(k)\|u\|, k \in I\right\} .
$$

Using (9), we have that $L(K) \subset K_{2}$. Moreover, $v=\frac{1}{r(L)} L v \in K_{2}$.

Let $\widetilde{u}(k)=\sum_{s=a}^{b} G(k, s)\left(a_{1}+b_{1} \Phi\left(u_{2}\right)+c_{2}\right)$. Then, for all $k \in I$, the following inequalities hold:

$$
\widetilde{u}(k) \leq \sum_{s=a}^{b} G(k, s)\left(c_{3}+b_{1} \varepsilon_{3}\left|u_{2}\right|+b_{1} \Phi^{*}\right) \leq l_{1}(k)\left(c_{3}+b_{1} \varepsilon_{3} R+b_{1} \Phi^{*}\right) \sum_{s=a}^{b} \varphi(s) .
$$

Therefore,

$$
\|\tilde{u}\| \leq \max _{k \in I} l_{1}(k)\left(c_{3}+b_{1} \varepsilon_{3} R+b_{1} \Phi^{*}\right) \sum_{s=a}^{b} \varphi(s) .
$$

Note that from the definition of $R$, since $R>R_{1}$, we have $\|\tilde{u}\|<R$.

Assume, on the contrary, that there exist $u_{2} \in \partial B_{R}$ and $\mu_{2}>0$ such that

$$
u_{2}-T u_{2}=\mu_{2} v
$$

From

$$
\begin{aligned}
u_{2}(k)+\widetilde{u}(k) & =\widetilde{u}(k)+\left(T u_{2}\right)(k)+\mu_{2} v(k) \\
& =\sum_{s=a}^{b} G(k, s)\left(f\left(s, u_{2}(s)\right)+b_{1} \Phi\left(u_{2}(s)\right)+a_{1}+c_{2}\right)+\mu_{2} v(k),
\end{aligned}
$$

using (H3), $L(K) \subset K_{2}$, and $v \in K_{2}$, we deduce that $u_{2}+\widetilde{u} \in K_{2}$.

As a result, we obtain that, for all $k \in I$,

$$
\begin{aligned}
\widetilde{u}(k)+\left(T u_{2}\right)(k) & =\sum_{s=a}^{b} G(k, s)\left(f\left(s, u_{2}(s)\right)+b_{1} \Phi\left(u_{2}(s)\right)+c_{3}\right) \\
& \geq \sum_{s=a}^{b} G(k, s)\left(\left(\frac{1}{r(L)}+\varepsilon_{2}-b_{1} \varepsilon_{3}\right)\left|u_{2}(s)\right|-c_{3}+b_{1} \Phi\left(u_{2}(s)\right)+c_{3}\right) \\
& \geq\left(\frac{1}{r(L)}+\varepsilon_{2}-b_{1} \varepsilon_{3}\right) \sum_{s=a}^{b} G(k, s)\left|u_{2}(s)\right| \\
& \geq\left(\frac{1}{r(L)}+\varepsilon_{2}-b_{1} \varepsilon_{3}\right) \sum_{s=a}^{b} G(k, s) u_{2}(s) .
\end{aligned}
$$

Now, we claim that

$$
\left(\varepsilon_{2}-b_{1} \varepsilon_{3}\right) \sum_{s=a}^{b} G(k, s) u_{2}(s) \geq \frac{1}{r(L)} \sum_{s=a}^{b} G(k, s) \widetilde{u}(s),
$$


which is equivalent to

$$
\left(\varepsilon_{2}-b_{1} \varepsilon_{3}\right) \sum_{s=a}^{b} G(k, s)\left(u_{2}(s)+\widetilde{u}(s)\right) \geq\left(\frac{1}{r(L)}+\varepsilon_{2}-b_{1} \varepsilon_{3}\right) \sum_{s=a}^{b} G(k, s) \widetilde{u}(s) .
$$

Since $u_{2}+\tilde{u} \in K_{2}$, we have that $u_{2}(k)+\widetilde{u}(k) \geq l_{1}(k)\left\|u_{2}+\widetilde{u}\right\| \geq l_{1}(k)\left(\left\|u_{2}\right\|-\|\widetilde{u}\|\right)$. Then

$$
\left(\varepsilon_{2}-b_{1} \varepsilon_{3}\right) \sum_{s=a}^{b} G(k, s)\left(u_{2}(s)+\widetilde{u}(s)\right) \geq\left(\varepsilon_{2}-b_{1} \varepsilon_{3}\right)(R-\|\widetilde{u}\|) \sum_{s=a}^{b} G(k, s) l_{1}(s) .
$$

From (17) and from the definition of $R$, since $R>R_{2}$, we deduce

$$
\begin{aligned}
\left(\varepsilon_{2}-b_{1} \varepsilon_{3}\right)(R-\|\widetilde{u}\|) & \geq\left(\varepsilon_{2}-b_{1} \varepsilon_{3}\right)\left(R-\max _{k \in I} l_{1}(k)\left(c_{3}+b_{1} \varepsilon_{3} R+b_{1} \Phi^{*}\right) \sum_{s=a}^{b} \varphi(s)\right) \\
& \geq\left(\frac{1}{r(L)}+\varepsilon_{2}-b_{1} \varepsilon_{3}\right)\left(c_{3}+b_{1} \varepsilon_{3} R+b_{1} \Phi^{*}\right) \sum_{s=a}^{b} \varphi(s) .
\end{aligned}
$$

Finally, from (16) we obtain

$$
\begin{aligned}
& \left(\varepsilon_{2}-b_{1} \varepsilon_{3}\right) \sum_{s=a}^{b} G(k, s)\left(u_{2}(s)+\widetilde{u}(s)\right) \\
& \geq\left(\varepsilon_{2}-b_{1} \varepsilon_{3}\right)(R-\|\widetilde{u}\|) \sum_{s=a}^{b} G(k, s) l_{1}(s) \\
& \geq\left(\frac{1}{r(L)}+\varepsilon_{2}-b_{1} \varepsilon_{3}\right)\left(c_{3}+b_{1} \varepsilon_{3} R+b_{1} \Phi^{*}\right) \sum_{s=a}^{b} G(k, s) l_{1}(s) \sum_{j=a}^{b} \varphi(j) \\
& \geq\left(\frac{1}{r(L)}+\varepsilon_{2}-b_{1} \varepsilon_{3}\right) \sum_{s=a}^{b} G(k, s) \widetilde{u}(s),
\end{aligned}
$$

which proves our claim.

Now, using (20) it follows that

$$
\begin{aligned}
\left(\frac{1}{r(L)}+\varepsilon_{2}-b \varepsilon_{3}\right) \sum_{s=a}^{b} G(k, s) u_{2}(s)= & \frac{1}{r(L)} \sum_{s=a}^{b} G(k, s)\left(u_{2}(s)+\widetilde{u}(s)\right) \\
& -\frac{1}{r(L)} \sum_{s=a}^{b} G(k, s) \widetilde{u}(s) \\
& +\left(\varepsilon_{2}-b_{1} \varepsilon_{3}\right) \sum_{s=a}^{b} G(k, s) u_{2}(s) \\
\geq & \frac{1}{r(L)} \sum_{s=a}^{b} G(k, s)\left(u_{2}(s)+\widetilde{u}(s)\right) .
\end{aligned}
$$


From (19) and (21), we deduce

$$
\left(T u_{2}\right)(k)+\widetilde{u}(k) \geq \frac{1}{r(L)} \sum_{s=a}^{b} G(k, s)\left(u_{2}(s)+\widetilde{u}(s)\right)=\frac{\left(L\left(u_{2}+\widetilde{u}\right)\right)(s)}{r(L)}
$$

Then (18) gives us

$$
u_{2}+\widetilde{u}=T u_{2}+\widetilde{u}+\mu_{2} v \geq \frac{L\left(u_{2}+\widetilde{u}\right)}{r(L)}+\mu_{2} v \geq \mu_{2} v
$$

Define $\mu^{*}:=\sup \left\{\mu>0 \mid u_{2}+\tilde{u} \geq \mu \nu\right\}$. Note that $\mu_{2} \in\left\{\mu>0 \mid u_{2}+\tilde{u} \geq \mu \nu\right\}$. Thus $\mu^{*} \geq \mu_{2}$ and $u_{2}+\tilde{u} \geq \mu^{*} v$. Using (5) we deduce that

$$
\frac{L\left(u_{2}+\widetilde{u}\right)}{r(L)} \geq \frac{\mu^{*}}{r(L)} L v=\mu^{*} v
$$

whence

$$
u_{2}+\tilde{u} \geq\left(\mu_{2}+\mu^{*}\right) v
$$

which contradicts the definition of $\mu^{*}$.

Then (15) holds and Lemma 3 gives us that $\operatorname{deg}\left(I-T, B_{R}, 0\right)=0$.

On the other hand, from $(H 6)$, there exist $\varepsilon_{4} \in\left(0, \frac{1}{r(L)}\right)$ and $r \in(0, R)$ such that

$$
|f(k, u)| \leq\left(\frac{1}{r(L)}-\varepsilon_{4}\right)|u| \quad \text { for all } k \in I \text { and }|u|<r \text {. }
$$

We will prove that

$$
T u \neq \mu u \quad \text { for every } u \in \partial B_{r} \text { and } \mu \geq 1 \text {. }
$$

Assume on the contrary that there exist $u_{3} \in B_{r}$ and $\mu_{3} \geq 1$ such that $T u_{3}=\mu_{3} u_{3}$. Then

$$
\begin{aligned}
\left|u_{3}(k)\right| & =\frac{1}{\mu_{3}}\left|\left(T u_{3}\right)(k)\right| \leq\left|\left(T u_{3}\right)(k)\right|=\left|\sum_{s=a}^{b} G(k, s) f\left(s, u_{3}(s)\right)\right| \\
& \leq \sum_{s=a}^{b} G(k, s)\left|f\left(s, u_{3}(s)\right)\right| \leq\left(\frac{1}{r(L)}-\varepsilon_{4}\right) \sum_{s=a}^{b} G(k, s)\left|u_{3}(s)\right|
\end{aligned}
$$

and $\left(I-\left(\frac{1}{r(L)}-\varepsilon_{4}\right) L\right)\left|u_{3}\right| \leq 0$.

So, arguing as in the proof of Theorem 5, with $\alpha=\frac{1}{r(L)}-\varepsilon_{4}$, we deduce that $u_{3}(k) \equiv 0$, in contradiction with $u_{3} \in \partial B_{r}$. Hence (22) holds and from Lemma 4 we deduce

$$
\operatorname{deg}\left(I-T, B_{r}, 0\right)=1
$$

Thus,

$$
\operatorname{deg}\left(I-T, B_{R} \backslash \overline{B_{r}}, 0\right)=\operatorname{deg}\left(I-T, B_{R}, 0\right)-\operatorname{deg}\left(I-T, B_{r}, 0\right)=-1 .
$$


Therefore, operator $T$ has at least one fixed point in $B_{R} \backslash \overline{B_{r}}$, which is a nontrivial solution of (2).

\section{Examples}

In this section we introduce a few examples where the applicability of the existence results proved in the previous section is pointed out.

Example 7 Consider the second order problem with Dirichlet conditions

$$
\left\{\begin{array}{l}
-\Delta^{2} u(k-1)=f(k, u(k)), \quad k \in J_{1}:=\{1, \ldots, N-1\} \\
u(0)=0=u(N)
\end{array}\right.
$$

We have that this problem is equivalent to the operator equation

$$
T u(k)=\sum_{s=1}^{N-1} G(k, s) f(s, u(s))=u(k) \quad \text { for } k=\{0,1, \ldots, N\}
$$

with

$$
G(k, s)=\frac{1}{N} \begin{cases}(N-s) k, & 0 \leq k \leq s \leq N-1, \\ (N-k) s, & 1 \leq s \leq k \leq N\end{cases}
$$

Clearly, $G(0, s)=0=G(N, s)$ for all $s \in J_{1}$ and $G(k, s)=G(s, k)>0$ for all $(k, s) \in J_{1} \times J_{1}$.

If we restrict the equation to the interval $J_{1}$ and set the operator

$$
\widetilde{T} u(k)=\sum_{s=1}^{N-1} G(k, s) f(s, u(s)) \quad \text { for } k \in J_{1},
$$

we have that the Green's function $G(k, s)$ satisfies conditions $(G 1)-(G 3)$ on $J_{1} \times J_{1}$.

Moreover, the smallest eigenvalue of the eigenproblem

$$
\left\{\begin{array}{l}
-\Delta^{2} u(k-1)=\lambda u(k), \quad k \in J_{1} \\
u(0)=0=u(N)
\end{array}\right.
$$

is $\lambda_{1}=4 \sin ^{2} \frac{\pi}{2 N}$ and $r_{1}(L)=\frac{1}{\lambda_{1}}=\frac{1}{4 \sin ^{2} \frac{\pi}{2 N}}$.

Similar results for the same second order problem with periodic conditions $u(0)=u(N)$, $u(1)=u(N+1)$ or with Neumann conditions $u(0)=u(N), \Delta u(0)=\Delta u(N)$ might be obtained.

Example 8 Now, consider the fourth order Lidstone problem

$$
\left\{\begin{array}{l}
\Delta^{4} u(k-2)+\Delta^{2} u(k-1)=f(k, u(k)), \quad k \in J_{2}:=\{2, \ldots, N+1\} \\
u(1)=\Delta^{2} u(0)=u(N+1)=\Delta^{2} u(N)=0 .
\end{array}\right.
$$

It is shown in [14] that the corresponding Green's function $G(k, s)$ is strictly positive and symmetric on $J_{2} \times J_{2}$ and it also satisfies conditions (G1)-(G3). 
One can compute that in this case

$$
\lambda_{2}=16 \sin ^{4} \frac{\pi}{2 N}+4 \sin ^{2} \frac{\pi}{2 N},
$$

whence

$$
r_{2}(L)=\frac{1}{\lambda_{2}}=\frac{1}{16 \sin ^{4} \frac{\pi}{2 N}+4 \sin ^{2} \frac{\pi}{2 N}} .
$$

Example 9 Consider now the fourth order Lidstone problem

$$
\left\{\begin{array}{l}
\Delta^{4} u(k-2)=f(k, u(k)), \quad k \in J_{3}:=\{2, \ldots, N+1\}, \\
u(1)=\Delta^{2} u(0)=u(N+1)=\Delta^{2} u(N)=0 .
\end{array}\right.
$$

One can verify (see $[15,18]$ ) that the corresponding Green's function $G(k, s)$ is strictly positive and symmetric on $J_{3} \times J_{3}$ and it also satisfies conditions (G1)-(G3). Moreover,

$$
r_{3}(L)=\frac{1}{16 \sin ^{4} \frac{\pi}{2 N}} .
$$

As a direct consequence, we have that the enunciations of Theorems 5 and 6 for this problem coincide, respectively, with Theorems 3.2 and 3.1 in [18].

Example 10 Consider the inverse difference equation

$$
u(k)=\sum_{s=1}^{N-1} f(s, u(s)) \quad \text { for } k \in J_{4}:=\{1, \ldots, N-1\}
$$

which corresponds to the kernel

$$
G(k, s)=1, \quad k, s \in J_{4} .
$$

It is immediate to verify that the eigenvalues of the matrix function $G(k, s)$ are $N-1$ and 0 . So $r_{4}(L)=N-1$.

Example 11 Let $N=101$ and consider the inverse difference equation

$$
u(k)=\sum_{s=1}^{100}(k+s)^{2} f(s, u(s)) \quad \text { for } k \in J_{5}:=\{1, \ldots, 100\}
$$

which corresponds to the kernel

$$
G(k, s)=(k+s)^{2}, \quad k, s \in J_{5}
$$

By numerical approach, one may verify that the nonzero eigenvalues of $G$ are given by $1.45456 \times 10^{6},-107,101$, and 5940.08 . In consequence,

$$
r_{5}(L) \approx 1.45456 \times 10^{6}
$$


For any of the previous examples, we can take

$$
f(t, x)=p\left|\sin x+\frac{x^{3}}{x^{2}+1}\right|-t q\left(1-e^{-x}\right), \quad(t, x) \in I \times \mathbb{R},
$$

with $p>\frac{1}{r_{i}(L)}>|p-t q|$ for $i=1,2,3,4$ and $t \in I$.

Since

$$
\lim _{|x| \rightarrow 0} \frac{|f(t, x)|}{|x|}=|p-t q| \text { and } \lim _{|x| \rightarrow \infty} \frac{f(t, x)}{|x|}=p,
$$

it is clear that conditions $(H 0)$ and $(H 3)-(H 6)$ hold.

We notice that a necessary condition on $p$ and $q$ is

$$
0<q<\frac{2}{N-1} p
$$

From Theorem 6 it follows that all of the considered examples have at least one nontrivial solution.

Moreover, choose

$$
f(t, x)= \begin{cases}t p\left(\sin x+\frac{x^{3}}{x^{2}+1}\right)+q \tanh x, & x \geq 0, \\ t\left(p\left(\sin x+\frac{x^{3}}{x^{2}+1}\right)-q \frac{2^{x}-1}{\ln 2}\right), & x \leq 0\end{cases}
$$

with

$$
\frac{1}{r_{i}(L)}-q<p<\frac{1}{r_{i}(L)(N-1)}
$$

Due to the fact that

$$
\lim _{x \rightarrow 0^{+}} \frac{f(t, x)}{x}=t p+q, \quad \lim _{x \rightarrow 0^{-}} \frac{f(t, x)}{x}=t(p-q), \quad \text { and } \quad \lim _{|x| \rightarrow \infty} \frac{|f(t, x)|}{|x|}=t p,
$$

we deduce that conditions $(H 0)-(H 2)$ hold and, from Theorem 5 , we have that all of the considered examples have at least one nontrivial solution.

Acknowledgements

Not applicable.

Funding

First author is partially supported by Xunta de Galicia (Spain), project EM2014/032 and AIE, Spain and FEDER, grant MTM2016-75140-P. The second author is supported by the Bulgarian National Science Foundation under Project DN 12/4 "Advanced analytical and numerical methods for nonlinear differential equations with applications in finance and environmental pollution".

Availability of data and materials

The data sets used and analyzed during the current study are available from the corresponding author on reasonable request.

Ethics approval and consent to participate

Not applicable. 


\section{Consent for publication}

The authors confirm that the work described has not been published before (except in the form of an abstract or as part of a published lecture, review, or thesis), and that its publication has been approved by all co-authors.

\section{Authors' contributions}

All authors have contributed equally to this manuscript. All authors read and approved the final manuscript.

\section{Authors' information}

Not applicable.

\section{Author details}

'Departamento de Estatística, Análise Matemática e Optimización, Instituto de Matemáticas, Facultade de Matemáticas, Universidade de Santiago de Compostela, Santiago de Compostela, Spain. ${ }^{2}$ Department of Mathematics, University of Ruse, Ruse, Bulgaria.

\section{Publisher's Note}

Springer Nature remains neutral with regard to jurisdictional claims in published maps and institutional affiliations.

Received: 28 May 2019 Accepted: 17 October 2019 Published online: 25 October 2019

\section{References}

1. Agarwal, R.P.: Difference Equations and Inequalities. Theory, Methods, and Applications, 2nd edn. Monographs and Textbooks in Pure and Applied Mathematics, vol. 228. Dekker, New York (2000)

2. Anuradha, V., Hai, D.D., Shivaji, R.: Existence results for superlinear semipositone BVP's. Proc. Am. Math. Soc. 124(3) 757-763 (1996)

3. Bai, D., Henderson, J., Zeng, Y.: Positive solutions of discrete Neumann boundary value problems with sign-changing nonlinearities. Bound. Value Probl. 2015, 231 (2015). https://doi.org/10.1186/s13661-015-0500-8

4. Bai, D., Xu, Y.: Positive solutions for semipositone BVPs of second-order difference equations. Indian J. Pure Appl. Math. 39, 59-68 (2008)

5. Cabada, A., Dimitrov, N.: Multiplicity results for nonlinear periodic fourth order difference equations with parameter dependence and singularities. J. Math. Anal. Appl. 371(2), 518-533 (2010)

6. Cabada, A., Ferreiro, J.B.: Existence of positive solutions for $n$ th-order periodic difference equations. J. Differ. Equ. Appl. 17(6), 935-954 (2011)

7. Cabada, A., Otero-Espinar, V.: Optimal existence results for $n$th order periodic boundary value difference equations. J. Math. Anal. Appl. 247(1), 67-86 (2000)

8. Cabada, A., Otero-Espinar, V.: Fixed sign solutions of second-order difference equations with Neumann boundary conditions. Advances in difference equations, IV. Comput. Math. Appl. 45(6-9), 1125-1136 (2003)

9. Dahal, R., Duncan, D., Goodrich, C.S.: Systems of semipositone discrete fractional boundary value problems. J. Differ. Equ. Appl. 20(3), 473-491 (2014)

10. Deimling, K.: Nonlinear Functional Analysis. Springer, Berlin (1985)

11. Goodrich, C.S.: On a first-order semipositone discrete fractional boundary value problem. Arch. Math. 99, 509-518 (2012)

12. Goodrich, C.S.: On semipositone discrete fractional boundary value problems with non-local boundary conditions. J. Differ. Equ. Appl., 19(11), 1758-1780 (2013)

13. Guo, D.J., Lakshmikantham, V.: Nonlinear Problems in Abstract Cones. Notes and Reports in Mathematics in Science and Engineering, vol. 5. Academic Press, Boston (1988)

14. $\mathrm{He}, \mathrm{T}$., Su, Y.: On discrete fourth-order boundary value problems with three parameters. J. Comput. Appl. Math. 233(10), 2506-2520 (2009)

15. $\mathrm{Xu}, \mathrm{J}$.: Positive solutions for a fourth order discrete $\mathrm{p}$-Laplacian boundary value problem. Math. Methods Appl. Sci. 36(18), 2467-2475 (2013)

16. Zeidler, E.: Nonlinear Functional Analysis and Its Applications. I. Fixed-Point Theorems. Springer, New York (1986)

17. Zhang, K.: Nontrivial solutions of fourth-order singular boundary value problems with sign-changing nonlinear terms. Topol. Methods Nonlinear Anal. 40(1), 53-70 (2012)

18. Zhang, K., O'Regan, D., Fu, Z.: Nontrivial solutions for boundary value problems of a fourth order difference equation with sign-changing nonlinearity. Adv. Differ. Equ. 2018, 370 (2018). https://doi.org/10.1186/s13662-018-1840-3 